\title{
Exudative Retinal Detachment Associated with Complicated Retrobulbar Anesthesia
}

\author{
Volkan Yaylalı1,2, Ibrahim Toprak ${ }^{3 *}$ \\ ${ }^{1}$ Department of Ophthalmology, Faculty of Medicine, Pamukkale University, Denizli, Turkey \\ ${ }^{2}$ Private Yaylalı Eye Hospital, Denizli, Turkey \\ ${ }^{3}$ Department of Ophthalmology, Servergazi State Hospital, Denizli, Turkey \\ Email: volkanyaylali@yahoo.com, ${ }^{*}$ ibrahimt@doctor.com
}

Received 11 January 2015; accepted 31 January 2015; published 3 February 2015

Copyright (C) 2015 by authors and Scientific Research Publishing Inc.

This work is licensed under the Creative Commons Attribution International License (CC BY). http://creativecommons.org/licenses/by/4.0/

cc) (i) Open Access

\begin{abstract}
Retrobulbar anesthesia (block) is used for many ocular surgeries, whereas it is well known that this procedure has complications such as for retrobulbar hemorrhage, globe perforation, optic nerve injury and brain stem anesthesia. In this report, we present a unique case in the literature of isolated exudative retinal detachment (RD) secondary to iatrogenic retrobulbar hemorrhage. A 73-year-old woman underwent retrobulbar block for combined phaco-vitrectomy. Immediately after the injection, progressive proptosis was recognized. The globe was decompressed and she underwent combined phaco-vitrectomy after stabilization of the eye on the same day. At the beginning of the vitrectomy, a dome shaped serous RD was observed in the infero-temporal quadrant. Peripheral exploration was performed, whereas there was no retinal tear or hole. On the first day postoperatively, serous RD was disappeared. In conclusion, this report suggests that increased intraorbital pressure secondary to iatrogenic retrobulbar hemorrhage might lead exudative RD.
\end{abstract}

\section{Keywords}

Retrobulbar Anesthesia, Retrobulbar Hemorrhage, Exudative Retinal Detachment

\section{Introduction}

Topical anesthesia is widely used for elective phaco surgery, whereas in complicated cases and posterior segment surgeries, surgeons prefer retrobulbar block, which provides a deeper anesthesia [1]. Injection of local anesthetic agents into retrobulbar space carries risks for many local and systemic complications such as subcon-

\footnotetext{
*Corresponding author.
} 
junctival hemorrhage, chemosis, retrobulbar hemorrhage, extraocular muscle injury, globe perforation, optic neuropathy, retinal artery occlusion, seizures, transient blindness, cardiopulmonary collapse, III cranial nerve palsy and brainstem anesthesia [1]-[7].

Retrobulbar hemorrhage is a relatively common complication of retrobulbar block with an incidence of $0.1 \%$ - 3\% [1] [2]. The sharp needle causes vascular injury and bleeding, which results in progressive proptosis, elevated intraocular pressure (IOP), chemosis and swelling of the eyelids. Immediate IOP lowering therapy such as intravenous mannitol infusion and lateral canthotomy are generally needed to prevent permanent visual damage [1] [2]. To best of our knowledge, this is the first study to report a case with isolated exudative retinal detachment following retrobulbar hemorrhage as a complication of retrobulbar block.

\section{Case Report}

A 73-year-old woman presented with visual deterioration in both eyes. A complete ophthalmological examination was performed. Best-corrected visual acuity (BCVA) was 0.6 and 0.2 (Snellen chart) in the right and left eyes (respectively). Slit-lamp biomicroscopy and dilated fundoscopy revealed bilateral senile cataract and epimacular membrane in the left eye. The patient was informed and combined phacoemulsification and pars plana vitrectomy (PPV) surgery was planned for the left eye.

On the operation day, the patient underwent retrobulbar anesthesia and three milliliters of local anesthetic agent was injected into the left retrobulbar space using Atkinson needle (25-gauge $\times 32 \mathrm{~mm}$ ) at inferolateral orbital region as commonly described [1]. Immediately after the injection, dramatic proptosis, chemosis and eyelid edema were observed. Lateral canthotomy was performed urgently and intravenous mannitol infusion (300 cc 20\%) was administered. The patient had no signs of systemic complications of retrobulbar block. Arterial blood pressure, heart rate, electrocardiography and pulse oximetry values were within normal limits. In an hour, marked proptosis and eyelid edema regressed, and IOP returned to normal. Phacoemulsification and 23-gauge PPV were performed in the same session.

At the beginning of the vitrectomy, a localized, dome shaped serous retinal detachment (RD) with a smooth surface was observed in the infero-temporal quadrant incidentally, which was not present in preoperative fundus examination (Figure 1). Peripheral retinal exploration was performed under scleral indentation, whereas we found no retinal break or signs of globe perforation. The operation was completed without any complication. On the first day postoperatively, dilated fundus examination revealed no serous retinal detachment or retinal tear in the left eye. During the postoperative follow up, ocular examinations were non-specific and the patient had a BCVA of 0.8 (Snellen) in the left eye.

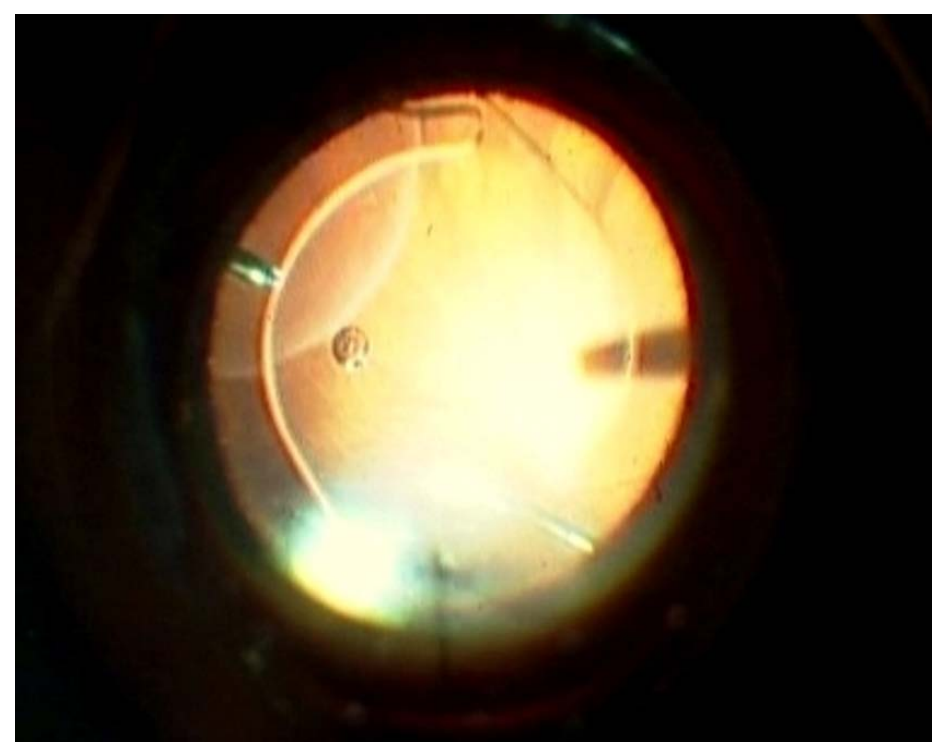

Figure 1. Intraoperative fundus image demonstrates infero-temporal quadrant located and dome shaped exudative retinal detachment (this image was captured from intraoperative video record and loss of resolution is related with intraoperative lighting conditions). 


\section{Discussion}

The current literature comprises cases with common complications of retrobulbar block and RD related to iatrogenic globe perforation. However, there are a few numbers of case-reports presenting localized RD as a complication of retrobulbar anesthesia. Mieler et al. [2] presented a case with localized RD and coexisting central retinal artery and vein occlusion due to possible injection of the anesthetic agent into the optic nerve [2]. They reported that the patient showed no acute neurological symptoms, whereas visual loss was severe and permanent. Another study by Mameletzi et al. [8] reported a 78-year-old female with severe vision loss (light perception), multiple retinal arterial emboli, localized RD and magnetic resonance imaging (MRI) findings of optic neuritis in her left eye a day after uneventful cataract surgery, which was performed under retrobulbar anesthesia. They reported that retina was reattached after administration of IOP lowering therapy and intravenous methylprednisolone (1 g/day for three days), whereas visual acuity did not change [8].

In our study, isolated exudative RD might be due to compression of ocular venous drainage system like a vortex vein by retrobulbar hematoma and elevated intraorbital pressure [9] [10]. Under normal conditions, fluid is transported from vitreous to the choroid by active pumping function of retinal pigment epithelium and hydrostatic gradient. Deterioration in inflow-outflow balance leads to accumulation of fluid in the subretinal area and exudative RD develops [9] [10].

Herein, retrobulbar hematoma or/and increased intraorbital pressure might cause obstruction of venous outflow. However, quadrantic localization of the exudative RD might suggest vortex vein compression or damage as in nanophthalmic eyes and eyes underwent scleral buckling surgery [9] [10]. Rapid spontaneous resolution of exudative RD postoperatively can be explained with normalization of the intraorbital pressure.

\section{Conclusion}

In conclusion, our study might add exudative RD into the current literature as a complication of retrobulbar block.

\section{Acknowledgements}

This study was performed in adherence to the tenets of the Declaration of Helsinki. No author has a financial or proprietary interest in any product, material, or method mentioned. No financial support was received for this study.

\section{References}

[1] Jaichandran, V. (2013) Ophthalmic Regional Anaesthesia: A Review and Update. Indian Journal of Anaesthesia, 3, 7-13. http://dx.doi.org/10.4103/0019-5049.108552

[2] Mieler, W.F., Bennett, S.R., Platt, L.W. and Koenig, S.B. (1990) Localized Retinal Detachment with Combined Central Retinal Artery and Vein Occlusion after Retrobulbar Anesthesia. Retina, 10, 278-283. http://dx.doi.org/10.1097/00006982-199010000-00010

[3] Tappeiner, C. and Garweg, J.G. (2011) Retinal Vascular Occlusion after Vitrectomy with Retrobulbar AnesthesiaObservational Case Series and Survey of Literature. Graefe's Archive for Clinical and Experimental Ophthalmology, 249, 1831-1815. http://dx.doi.org/10.1007/s00417-011-1783-9

[4] Gross, A. and Cestari, D.M. (2014) Optic Neuropathy Following Retrobulbar İnjection: A Review. Seminars in Ophthalmology, 29, 434-439. http://dx.doi.org/10.3109/08820538.2014.959191

[5] Naik, A.A., Agrawal, S.A., Navadiya, I.D. and Ramchandani, S.J. (2014) Management of Macular Epiretinal Membrane Secondary to Accidental Globe Perforation during Retrobulbar Anesthesia. Indian Journal of Ophthalmology, 62, 94-95.

[6] Spire, M., Fleury, J., Kodjikian, L. and Grange, J.D. (2007) Retinal Detachment Caused by Ocular Perforation during Periocular Anesthesia: Three Case Reports. Journal Francais d'Ophtalmologie, 30, e16.

[7] Aranda Calleja, M.A., Martínez Pueyo, A., Bellido Cuellar, S. and García Ruiz, P. (2011) III Cranial Nerve Palsy and Brainstem Disfunction Following Retrobulbar Anaesthesia. Neurologia, 26, 563-564. http://dx.doi.org/10.1016/j.nrl.2011.04.013

[8] Mameletzi, E., Pournaras, J.A., Ambresin, A. and Nguyen, C. (2008) Retinal Embolisation with Localised Retinal Detachment Following Retrobulbar Anaesthesia. Klinische Monatsblätter für Augenheilkunde, 225, 476-478. http://dx.doi.org/10.1055/s-2008-1027268 
[9] Krohn, J. and Seland, J.H. (1998) Exudative Retinal Detachment in Nanophthalmos. Acta Ophthalmologica Scandinavica, 76, 499-502. http://dx.doi.org/10.1034/j.1600-0420.1998.760421.x

[10] Takahashi, K. and Kishi, S. (2000) Remodeling of Choroidal Venous Drainage after Vortex Vein Occlusion Following Scleral Buckling for Retinal Detachment. American Journal of Ophthalmology, 129, 191-198.

http://dx.doi.org/10.1016/S0002-9394(99)00425-0 
Scientific Research Publishing (SCIRP) is one of the largest Open Access journal publishers. It is currently publishing more than 200 open access, online, peer-reviewed journals covering a wide range of academic disciplines. SCIRP serves the worldwide academic communities and contributes to the progress and application of science with its publication.

Other selected journals from SCIRP are listed as below. Submit your manuscript to us via either submit@scirp.org or Online Submission Portal.
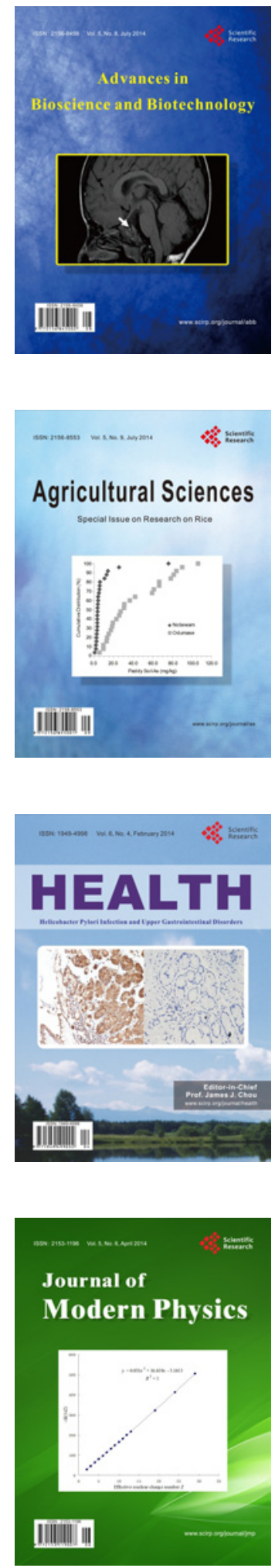
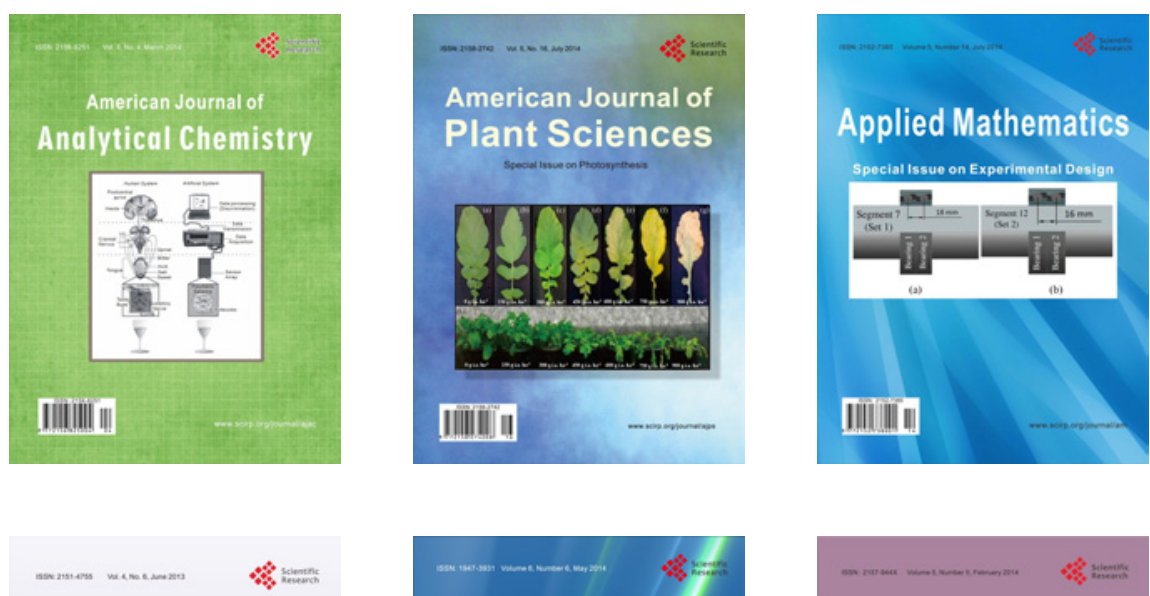

Creative Education
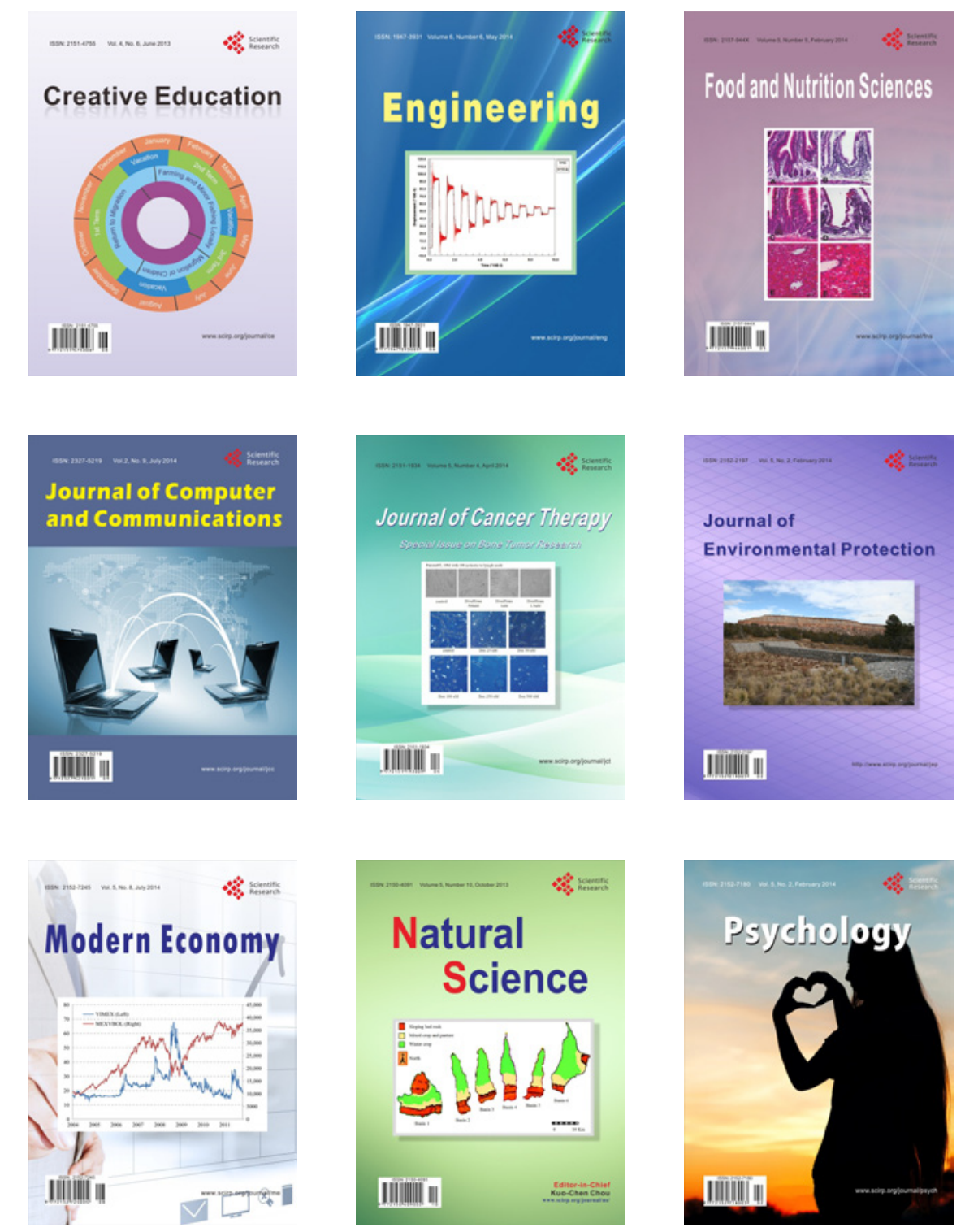